\title{
Perfil de preceptores e metodologias empregadas na formação de médicos residentes de um Hospital de Ensino do Estado do Pará
}

\author{
Profile of precepters and methodologies employed in \\ the training of physicians resident from a Hospital of \\ Teaching of the State of Pará
}

\author{
Vilma Francisca Hutim Gondim de Souza', Lizomar de Jesus Maués Pereira Moia ${ }^{2,3}$, \\ Ingrid Magali de Souza Pimentel ${ }^{1,3}$
}

'Fundação Casa de Misericórdia do Pará, Belém (PA), Brasil.

¿Universidade Federal do Pará, Belém (PA), Brasil.

3Universidade do Estado do Pará, Belém (PA), Brasil.

Recebido: Ago., 20, 2017 Aceito: Jun., 242019

\section{COMO CITAR ESTE ARTIGO}

Souza VFHG, Moia LJMP, Pimentel IMS Perfil de preceptores e metodologias empregadas na formação de médicos residentes de um Hospital de Ensino residentes de um Hospital de Ensino Journal of Health Education. 2018 Jan-Dez;3(1-2):24-32. https://doi. org/10.4322/ijhe.2018.002

\section{CORRESPONDÊNCIA}

Vilma Francisca Hutim Gondim de Souza

Fundação Casa de Misericórdia do Pará R. Bernal do Couto, 1040, Umarizal, CEP 66055-080, Belém (PA), Brasil Tel. (91) 98206-8086

vilmahutim@hotmail.com

\section{FONTE DE FINANCIAMENTO}

Nenhuma.

\section{CONFLITO DE INTERESSE}

Os autores declararam não

haver conflitos de interesse.

O estudo foi realizado na

Fundação Casa de Misericórdia

do Pará, Belém (PA), Brasil.

Todos os autores leram e aprovam a versão final submetida ao

Interdisciplinary Journal of Health Education (IJHE).

\section{RESUMO}

No atual contexto da medicina surgem novas demandas na atenção integral ao paciente, portanto, exigindo mudanças na formação dos profissionais de saúde. As metodologias inovadoras do processo de ensino e aprendizagem nos cursos da área saúde, no mundo, já são utilizadas há mais de 30 anos, embora, no Brasil, somente há mais de uma década. Na Residência Médica o processo ensino aprendizagem se baseia na transmissão de experiência do médico-preceptor para o residente, portanto, melhorias nesse processo requerem mudanças significativas na formação dos preceptores. Objetivo: caracterizar os preceptores dos programas de RM e conhecer as metodologias utilizadas nos cenários de prática, assim como seus conhecimentos sobre metodologias ativas. Método: foi desenvolvido um estudo exploratório de natureza qualitativa, por meio de entrevista semiestruturada. Foram abordados três eixos temáticos: I- caracterização dos preceptores; II- áreas de atuação, III-conhecimentos e práticas de metodologias de ensino, com ênfase no interesse desses preceptores em participar de qualificação pedagógica em metodologias ativas. A Análise de Conteúdo de Bardin foi usada para o estudo dos dados desta pesquisa. Resultados: a caracterização demográfica dos participantes foi: predominância do sexo feminino e faixa etária entre 50 e 60 anos nas oito Unidades de Registro (UR) abordadas. Todos os participantes verbalizaram a utilização da discussão de caso clínico real ou simulado para a busca do conhecimento e avaliação dos médicos residentes, embora oito UR relatem desconhecer as metodologias ativas de ensino aprendizagem. Doze (12) UR relatam conhecer essas metodologias e oito afirmam já utilizar algum método ativo na sua prática diária. Conclusão: analisando essas percepções, conclui-se que todos os preceptores entrevistados já utilizam algum método ativo, porém, intuitivamente, pois o residente é orientado a buscar o conhecimento teórico sobre os casos atendidos nos cenários da prática médica. Todos os participantes fizeram referência à necessidade de capacitação pedagógica e relataram que a ausência dessa prática acaba fragilizando o exercício adequado da preceptoria. Além dessa variável, todos os envolvidos citaram a infraestrutura institucional, garantia de educação permanente, plano de cargos, carreira e remuneração para preceptores como estratégias motivadoras e promotoras de qualidade no processo de ensino aprendizagem. A partir da trajetória teórica desse estudo, foi proposto um projeto de qualificação pedagógica em metodologias ativas para preceptores da RM da FSCMPA.

PALAVRAS-ChAVE: Preceptores. Residência médica. Formação de médicos.

\section{ABSTRACT}

In the current context of medicine, new demands around the full attention to the patient arise, thus requiring changes in the training of health professionals. Innovative methods of teaching and learning process in health courses in the world have already been used for over 30 years, although in Brazil only for more than a decade. In medical residency (MR), the learning process is based on the transmission of medical preceptor experience for the resident. Objective: so an improvement in this process requires significant changes in the training of preceptors. In order to know the methods and knowledge of the preceptors on methodologies and active references, in a teaching hospital, as well as their knowledge about active methods. Methods: it was developed an exploratory descriptive study with 
a qualitative nature, through semi-structured interviews. Three thematic axes were discussed: I- characterization of preceptors; II- fields of work, III-knowledge and teaching methods praxis, with emphasis on the interest of these preceptors to participate in pedagogical training in active methodologies. The content analysis under the perspective of Bardinwas used to study the data in this research. Results: The demographic characteristics of the participants were: predominantly female and aged between 50 and 60 years in the eight Registration Units (RU) addressed. All participants verbalized the use of real clinical case discussion or simulated for the pursuit of knowledge and evaluation of medical residents, although eight RU report not knowing the active methods of teaching and learning. Twelve (12) RU report they meet these methodologies and eight have reported the use of any active method in their daily practice. Conclusion: analyzing these perceptions, one may conclude that all interviewed preceptors have already used any active method, but in a intuitive way, since there's indents suggested to aquire theoretical knowledge aboutt he cases taken in the medical scenario in practice. All participants have mencioned the necessity of more pedagogical training and have also reported that the influence of this lake leads to a weakening of the right practice of preceptorism. Moreover, this variable, all involved cited the institutional infrastructure, continuing education guarantee, job plans, career and compensation for preceptors with motivating strategies and promoting quality in the teaching and learning process. From the theoretical trajectory of this study It was proposed a pedagogical training project in active methodologies for preceptors of MR of FSCMPA.

KEYWORDS: Preceptors. Medical residency. Medical training.

\section{Introdução}

A Residência Médica (RM),como pós-graduação, já tem mais de um século de existência e foi inicialmente implantada na América do Norte no Hospital John Hopkins nos Estados Unidos em 1848. Chegou ao Brasil em 1944, no Hospital de Clínicas coordenado pela Universidade de São Paulo. O segundo programa funcionou no Hospital dos Servidores no Rio de Janeiro em 1948, mas a RM só foi regulamentada pelo Decreto 80.281 de 5 de setembro de $1977^{1}$.

A função do preceptor tutor foi definida conforme a Resolução da Comissão Nacional de Residência Médica (CNRM) de n 005 de 8 de junho de 2004, com finalidade de orientar e oferecer diretrizes aos médicos residentes nos programas de treinamento, caracterizando o seu perfil como exigência de formação humanística e, também, ética. Foi definido como pré-requisito para sua atuação ter conhecimentos, habilidades e capacidade para desenvolver atividades didáticas durante a RM² .

A maioria dos programas de RM, no contexto brasileiro, ainda funciona sem institucionalização das regulamentações previstas pela CNRM, uma vez que foram reformulados os conteúdos na matriz curricular de várias áreas da medicina em resposta a demanda e necessidades de atender com mais qualidade e agilidade os serviços de saúde ${ }^{2}$.

Corroborando com Portal $\mathrm{MEC}^{2}$ a a prática diária dos profissionais de saúde demonstra que as competências técnicas, por si só, não conseguem atender a complexidade de atenção aos serviços de saúde, se considerada toda sua dimensão nas relações interdisciplinares e interpessoais que, na dinâmica diária, exige uma perspectiva ética e atitude humanística, além de demandar de capacidade de exercer liderança e gestão3.

Essa realidade exige quotidianamente que o preceptor desenvolva o papel de docente clínico, domine a prática clínica e também dos processos educacionais como metodologias e didáticas adequadas ao processo de ensino aprendizagem nos procedimentos do treinamento dos diversos programas de RM.

Ao analisar a formação oferecida nas escolas médicas do Brasil nos últimos 20 anos, verifica-se que a formação dos médicos ainda é deficitária, pois, apesar das mudanças nas diretrizes curriculares dos cursos de medicina, a maioria dos preceptores em atuação nos programas de RM não recebeu formação pedagógica para o ensino em saúde ${ }^{4}$.

A realização desse estudo abrolhou a partir da participação em capacitações pedagógicas para preceptores desenvolvidas no Estado do Pará, pelo Hospital Sírio Libanês/Instituto Sírio Libanês de Ensino e Pesquisa (IEP/HSL), Ministério da Saúde 
(MS)e ABEM em 2012, momento em que se construiu um projeto com proposta para capacitação pedagógica em metodologias ativas para preceptores dos programas de RM da FSCMPA, um hospital de ensino e referência estadual para maternidade de alto risco.

\section{Justificativa}

Na conjuntura brasileira, a RM tem como modalidade de pós-graduação o aperfeiçoamento das competências adquiridas na graduação, enfocando o desenvolvimento da capacidade de iniciativa, habilidades técnicas e metodológicas, bem como habilidade de julgamento e de avaliação, aguçando o desenvolvimento do espírito crítico e internalização de preceitos e normatizações éticas para o atendimento ao paciente em todos os níveis de assistência à saúde.

As legislações vigentes, a exemplo do Decreto 91.36421 de junho de 1985 pela criação da Comissão de Assessoria da Secretaria de Ensino Superior (SESu) do ministério da Educação responsáveis por planejar, orientar, coordenar e supervisionar os programas de Residências Médicas no Brasil, com todos atributos somados as iniciativas técnicas da ABEMCNRM, não conseguiram alcançar todas as regiões brasileiras, tão pouco produzir impacto na formação dos médicos dentro dos objetivos propostos ${ }^{4}$.

Pesquisas vêm sendo realizadas sobre os cursos de medicina com a finalidade de aperfeiçoar as metodologias realizadas na formação médica no Brasil. Tais estudos levam em consideração a demanda desses profissionais em atender as especificidades do trabalho e aprendizagem em saúde. É fundamental que nesse cenário haja a integração das esferas do ensino, orientação e serviço no processo de formação e atuação profissional, o que poderá fundamentalmente contribuir para ações interdisciplinares no cuidado em saúde ${ }^{5}$.

Segundo Skare ${ }^{6}$, o processo de ensino-aprendizagem nos programas de RM, na maioria de suas atividades operacionais, se desenvolve em torno de três dimensões essenciais: "O paciente, o residente e o preceptor", reforçando a necessidade de se considerar as especificidades inerentes a cada sujeito, a qual permeia tal relação. Portanto, deve-se ponderar e avaliar a influência das individualidades como visão de vida e de mundo, ressaltando a autoridade desse contexto na construção de todo este processo ${ }^{7}$.

Logo, um hospital de ensino, responsável pela formação de médicos residentes em várias áreas no estado, deve ter adequação regulamentada na CNRM e diretrizes da $\mathrm{ABEM}$ e DCN pertinentes à formação de profissionais mais críticos e reflexivos para atuar nos diversos níveis de atenção à saúde.

\section{Metodologia}

Foi realizado um estudo exploratório de natureza qualitativa a partir da descrição da percepção dos sujeitos nas respostas às questões das entrevistas utilizadas pelas autoras. Para isso, foi utilizado um aparelho celular da marca SAMSUNG GALAXY S4 mini através do gravador de voz para registro da entrevista e posterior transcrição pelas mesmas.

Utilizou-se entrevista semiestruturada com perguntas abertas e fechadas onde os participantes não tiveram identificação pessoal, sendo inferido um código identificador e nomeado como unidade de registro (UR) para citação da caracterização profissional.

Criou-se uma sigla para diferenciar a área de atuação na transcrição das falas dos participantes: CM01-médico da clínica médica, seguido do número indicativo de profissionais dessa área que foram entrevistados; MGO-médico da ginecologia obstetrícia; MP-médico pediatra; MCP-médico da cirurgia pediátrica; MCG-médico da cirurgia geral. 
Foram abordados três eixos de categorias: Questões sobre caracterização dos sujeitos do estudo e atuação do profissional; Questões e práticas de metodologias de ensino e metodologias ativas de ensino e aprendizagem; Questões sobre interesse $\backslash$ necessidade de capacitação em metodologias ativas de ensino e aprendizagem ${ }^{8,9}$.

Os participantes da pesquisa foram definidos a partir do cadastro de preceptores na Comissão de Residência Médica (COREME) da FSCMPA, os quais desenvolvem o papel de preceptoria de acordo com a resolução $\mathrm{CNRM}^{10}$. Foram entrevistados 20 preceptores dos 12 programas de RM, todos com vínculo institucional de 20 horas ou mais.

A coleta de dados iniciou-se a partir do registro do projeto de pesquisa na Plataforma Brasil, tendo todo o critério de obedecer às normatizações e preceitos da Declaração de Helsinque e do Código de Nuremberg e, atendendo as normas de pesquisa envolvendo seres humanos (Resolução CNS 466/12) do Conselho Nacional de Saúde. Foi submetido à aprovação do Comitê de Ética e Pesquisa da FSCMPA, parecer consubstanciado de $n^{\circ} 706.109$, aprovado em 24/06/2014. Ressalta-se que todo processo de coleta de dados foi realizado pelas autoras do estudo. A realização das entrevistas ocorreu de agosto a dezembro de 2014 e seu encerramento se deu por saturação amostral ${ }^{11}$.

Os resultados do estudo foram obtidos a partir da pré-análise e codificação do material, além do tratamento dos resultados, inferências e interpretações que nortearam as considerações finais do estudo em foco ${ }^{12}$.

\section{Resultados/discussões}

Para obtenção dos resultados e discussão das UR das entrevistas, foram analisadas três categorias, conforme mencionado anteriormente: Questões sobre caracterização dos sujeitos do estudo e atuação do profissional; Questões e práticas de metodologias de ensino e metodologias ativas de ensino e aprendizagem; Questões sobre interesse $\backslash$ necessidade de capacitação em metodologias ativas de ensino e aprendizagem.

\section{Caracterização e atuação profisisonal dos participantes da pesquisa}

De acordo coma a Tabela 1, podemos visualizar que em 11 UR predomina o sexo feminino. Observa-se também que, em oito UR, tem-se a faixa etária entre 51 e 60 anos, além de cinco UR com 41 a 50 anos. Todos os participantes possuem título de especialista, onde três UR com mestrado e dois com doutorado, ou seja, 15\% com essa titulação. 12 UR tem atuação exclusiva na FSCMPA e oito UR exercem docência em universidades.

Tabela 1. Distribuição dos participantes do estudo quanto ao sexo, faixa etária e o estado civil na FSCMPA no período de agosto a dezembro de 2014.

\begin{tabular}{lcc}
\multicolumn{1}{c}{ IDENTIFICAÇÃO } & FREQUÊNCIA & $\%$ \\
SEXO & 11 & 55,0 \\
Feminino & 9 & 45,0 \\
Masculino & & \\
\hline FAIXA ETÁRIA (EM ANOS) & 4 & 20 \\
$\leq 40$ & 5 & 25 \\
41 a 50 & 8 & 40 \\
51 a 60 & 3 & 15 \\
$>60$ & & 100 \\
\hline ESTADO CIVIL & 20 & \\
Casado(a) &
\end{tabular}

Fonte: Roteiro de entrevista aplicado pela autora. 
Quanto a área de atuação, a maioria dos participantes é da área da pediatria, totalizando 4 UR. Na ginecologia e obstetrícia tem-se três UR e na clínica médica e neonatologiaduas UR cada, onde todos distribuem-se nos 12 programas de RM da instituição.

O resultado da caracterização dos preceptores participantes do estudo de um hospital escola da região sudeste do Brasil que utilizou metodologia semelhante ao nosso estudo, apresentou caracterizações diferentes quanto ao sexo, idade e escolaridade dos preceptores participantes da pesquisa, ao que apresentou 16 preceptores com predomínio de faixa etária entre 40 a 49 anos de idade e predomínio do sexo masculino, além disso, $47 \%$ preceptores possuíam doutorado ${ }^{13}$.

\section{Questões sobre atuação profissional dos preceptores}

Aborda as percepções sobre o papel dos preceptores na formação dos médicos em nível de pós-graduação e o conhecimento das regulamentações da CNRM para o perfil dos médicos residentes e preceptores. Foram consideradas todas as contingências citadas ou sugestionadas como influentes e impactantes na relação dos três personagens do processo de ensino aprendizagem, a saber: preceptor, residente e paciente.

A maioria dos participantes referenciou como acontece o processo de ensino aprendizagem nesse cenário, ressaltando as condições de trabalho, valorização profissional, oportunidades de acesso e garantia de educação permanente, além do plano de cargos carreira e remuneração para o exercício da preceptoria. Muitas demandas vêm surgindo nesse processo de formação pela preceptoria, evidenciando a necessidade de integrar assistência, ensino e orientação em saúde ${ }^{5}$.

\section{[...] Acho que tem que integrar teoria e prática, assistência, ensino e serviço [...] (MCM01).}

Segundo estudo realizado em hospital de ensino na região sudeste do Brasil, os preceptores tiveram opiniões semelhantes às citadas por nossos participantes. Fizeram referência à falha na formação acadêmica, condições inadequadas de trabalho e falta de capacitação pedagógica para o exercício da preceptoria como contingências relacionadas às inadequações na formação do médico residente ${ }^{13}$.

A maioria dos participantes do estudo tem conhecimento do papel do preceptor, conforme está definido pela (CNRM) de $\mathrm{n}^{\circ} 005$ de 8 de junho de 2004, porém ressaltou quevárias contingências podem interferir no exercício adequado dessa função, como inadequação dos processos assistenciais e falha na gestão adequada dos serviços de saúde e falta de capacitação pedagógica.

[...] eu tenho observado aqui na instituição que o preceptor tem uma coisa intrínseca, pois eu não tenho uma formação acadêmica para exercer preceptoria [...] tem que ter um perfil de gostar de ensinar e passar o seu conhecimento [...] (MCP-01).

[...] sabidamente, a qualidade de um programa de RM está diretamente relacionada à qualidade de sua preceptoria [...] (MP-02).

Estudos apontam que, apesar das regulamentações do funcionamento da RM seguirem política de regulamentação, na prática, ainda existem diferenças regionais quanto à qualidade dos serviços que oferecem programas de RM sem garantias de preceptoria integral e capacitação pedagógica para essa função ${ }^{14}$.

[...] falta politica efetiva para o desenvolvimento da preceptoria tanto a nível nacional como institucional [...] (MG-02).

Neste cenário, ainda é necessário que haja valorização profissional dos preceptores no que diz respeito às condições de trabalho, plano de carreira, cargo e remuneração, assim, como garantia de educação permanente para os mesmos. 
A maior parte dos entrevistados ponderou problemas enfrentados como: alta demanda de pacientes em algumas áreas, falta de materiais para procedimentos nas áreas de cirurgias, principalmente na área de ginecologia e obstetrícia e na cirurgia geral. Em nível ambulatorial, foi relatado que a área física estaria insuficiente para atender aos pacientes de especialidades clínicas como reumatologia, clínica médica e ambulatório da mulher.

Redução do volume de atendimento para permitir um maior tempo dedicado ao estudo e ensino. Apoio financeiro para participação em congressos nacionais relacionados à atividade de ensino è è especialidade médica. Oferecimento de recursos físicos no ambiente de trabalho que facilitassem as atividades de ensino aprendizagem. Remuneração diferenciada que valorizasse a titulação, capacitação e tempo de serviço dedicado à preceptoria (MCM-02).

Segundo a Agência Nacional de Vigilância Sanitária (ANVISA), os procedimentos realizados em todos os níveis da assistência, desde a ambiência até os equipamentos adequados, constituem segurança do paciente ${ }^{15}$. A referência dos participantes do estudo é condizente com os resultados de publicações no caderno da ABEM sobre o tema em 2012 e outros estudos pilotos no Brasil ${ }^{14}$.

Conhecimentos e práticas de metodologias de ensino e metodologias ativas de ensino e aprendizagem

Observa-se nesta categoria que, a maioria dos entrevistados, relata a utilização de discussão de casos clínicos. Outros fazem discussão diária dos casos atendidos no momento da visita habitual aos pacientes internados, considerando que cada paciente atendido tem a discussão das condutas a serem definidas de acordo com a hipótese diagnóstica e o plano terapêutico discutido. Tais condutas têm como base as melhores evidências científicas e adaptações a protocolos clínicos para cada área de atuação. Além dessas atividades, todos os preceptores dizem seguir programações teóricas definidas pela matriz curricular de sua área específica.

Os preceptores fazem orientações embasadas na literatura e os residentes preparam as aulas que, na maioria das vezes, é expositiva para os residentes de áreas afins, ou seja, num ambiente fora do cenário de prática. Houve menção, ainda, a um cronograma de aulas semanais na COREME definida por programa.

[...] temos ensinado mais aulas expositivas e casos clínicos e como tecnicamente pode colocar em prática as teorias que embasam determinados procedimentos. Por falta de material fica mais na teoria (MGO-01).

\section{[...] utilizo mais casos clínicos, quando dá tempo (MGO-03).}

Alguns preceptores já utilizam metodologias objetivando que o aluno tenha uma busca mais ativa pelo conhecimento, embora sigam a matriz curricular preconizada pela CNRM vigente para cada área de atuação.

[...] vejo o residente muito afastado da busca ativa do conhecimento, ele está esperando aula, eu cobro que eles busquem conhecimento sobre os casos que atendemos no dia a dia no ambulatório (MGO-03).

O processo de avaliação dos médicos residentes, invariavelmente, segue as recomendações da matriz curricular na realização de provas teóricas elaboradas com respostas abertas e alternativas de múltipla escolha e uma média de três a seis provas escritas durante os dois anos na maioria dos programas de RM na instituição. Como complementação desse processo, é realizada por todos os programas a utilização de uma ficha padronizada pela COREME/FSCMPA que avalia habilidades cognitivas, interação pessoal, interesse e participação nas atividades assistenciais, dentre outras habilidades interativas. Segue a fala dos preceptores pertinentes a tal abordagem:

[...] sigo um protocolo de avaliação da COREME que avalia conhecimento da área, assiduidade, frequência, respeito e ética com o paciente, trabalhar em equipe, pontualidade, e atribuído um peso de o a 10 para essas categorias de avaliação, além disso, faço uma avaliação mensal (MGO-01). 
Quanto aos recursos utilizados para a avaliação dos médicos residentes, três UR fizeram referência à utilização básica das recomendações e diretrizes da CNRM/2006.

17 UR utilizavam metodologias alternativas na formação dos médicos residentes na FSCMPA, a exemplo da discussão de caso clínico com o objetivo de o residente realizar pesquisa de evidências sobre as patologias atendidas no cenário de prática.

Os preceptores, em sua maioria, relatam orientar o residente a fazer busca constante pelo conhecimento como instrumento técnico de avaliação somativa e também formativa, além de promover a discussão de caso em "sessões clínicas em forma de debate" como método de avaliar o residente através da participação, avaliando também a apresentação de conhecimento sobre a temática em discussão e entrega da pesquisa por escrito para fim documental e atribuição de conceito.

Além das atribuições definidas para os preceptores e residentes na CNRM, o regimento interno da FSCMPA acrescentou a complementação da carga horária e diversas atividades à matriz curricular, como a construção de um artigo científico para cada residente realizar seguindo a orientação de seus preceptores com o objetivo de incentivar a pesquisa na instituição.

Projetos de iniciação científica configuram-se como metodologia ativa, uma vez que a construção de artigo é norteada pela fase de elaboração do projeto, o qual requer que o aluno fundamentalmente busque conhecimentos sistemáticos e teóricos, desenvolvendo habilidades intelectuais em diversos níveis, desde a observação, descrição e análise até a argumentação da temática em estudo. Tal processo pode possibilitar novas iniciativas e maior segurança na tomada de decisões frente às demandas nos atendimentos em suas áreas de atuação ${ }^{16}$.

Segundo Guimarães ${ }^{17}$, os alunos podem desenvolver comportamentos intrinsecamente determinados para estabelecer metas e motivações pessoais, enfrentando os desafios diários da prática e aprendendo com o erro. Essa abordagem pode estabelecer capacidade de lidar com a frustração e provações. Apesar, de oito UR relatarem desconhecimento sistemático das metodologias ativas, todas utilizam a discussão e estudo de caso clínico nas suas práticas diárias, o que para muitos autores é considerada metodologia ativa de ensino aprendizagem ${ }^{18,19}$.

Desde a última década, no Brasil, os números de publicações sobre a inclusão das metodologias ativas na formação dos cursos na área da saúde, apontam que as novas demandas em saúde têm definido um novo perfil para o educador. Ele deve facilitar e orientar o aluno na busca pelo conhecimento e experiência num universo eclético que lhe confira mais autonomia como sujeito do processo de ensino aprendizagem ${ }^{20}$.

Pesquisa nacional fez avaliação de vantagens da utilização de metodologias ativas de ensino e aprendizagem na formação de estudantes de medicina e detectou que os alunos com dificuldade na sua formação tinham mais capacidade de lidar com as demandas sociais e afetivas dos pacientes ${ }^{7,21}$.

Interesse e necessidade de participar de capacitação em metodologias ativas de ensino e aprendizagem

Nessa categoria verifica-se que todos os participantes da pesquisa fazem referência à necessidade de capacitação pedagógica para preceptores e relacionam tal ação como mudança na condução do processo de ensino-aprendizagem nos programas de RM.

A inclusão do modelo de metodologias ativas de ensino e aprendizagem, com suas características de busca ativa pelo residente e facilitação da aprendizagem pelo preceptor, poderá contribuir para formação de profissionais mais sensíveis às condições socioeconômicas e culturais dos pacientes atendidos dos serviços saúde ${ }^{22}$.

[...] o que eu escuto no dia a dia dos colegas que atuam na instituição, a instituição não oferece reciclagem, capacitação e não oferece capacitação para essa função [...] (MP-04). 


\section{Considerações finais}

De acordo com as observações dos preceptores entrevistados, o modelo da preceptoria oferecido está correlacionado com as metodologias de ensino e aprendizagem pelas quais foram formados, bem como as capacitações que participaram ao longo do exercício profissional.

Observam-se, nas falas, falhas na efetivação das políticas que regulamentam o funcionamento dos programas de RM brasileira, falta de definição de papéis e perfil dos preceptores, políticas que estabeleçam plano de carreira e remuneração, além de garantias de acesso à educação permanente e continuada. Tais ressalvas foram citadas como causa de desmotivação no exercício da preceptoria.

A maioria dos entrevistados fez referência a metodologias ativas de ensino e aprendizagem, porém oito das 20 UR referem não utilizá-las nas suas práticas diárias.

Ao verificar a descrição das atividades diárias dos preceptores do estudo, constatou-se que todos desenvolvem, além de recomendações formais da CNRM, discussão de casos clínicos simulados ou reais a partir dos casos atendidos nos cenários de práticas e que os residentes são levados a buscar embasamento teórico para subsidiar as condutas clínicas adotadas no manejo de cada caso atendido. Essa dinâmica de ensino-aprendizagem integra as diversas modalidades de metodologias ativas, conforme descritas na justificativa do estudo.

A proposta de um projeto para capacitação pedagógica em metodologias ativas de ensino-aprendizagem para preceptores dos programas de RM da instituição, produto desse estudo, orientado pela equipe do Mestrado Profissional de Educação e Saúde da UEPA, veio atender às demandas expressas nas falas dos preceptores entrevistados.

\section{Referências}

1. Santos EG. Residência médica em cirurgia geral no Brasil - muito distante da realidade profissional. Rev Col Bras. 2009;36(3):271-76.

2. Brasil. Resolução CNRM No 005/2004, de 08 de junho de 2004. Dispõe sobre os serviços de preceptor/tutor dos programas de Residência Médica. Diário Oficial da União [Internet]. Brasília, 11 junho 2004 [citado em 2016 Maio 10]; Seção 1, p. 19. Disponível em: http://portal.mec.gov.br/sesu/arquivos/pdf/cnrm_052004.pdf

3. Brasil. Ministério da Educação. Resolução no 3, de 20 de Junho de 2014. Institui Diretrizes Curriculares Nacionais do Curso de Graduação em Medicina e dá outras providências. Diário Oficial da União, Brasília, 2014 [citado em 2019 Maio 15]; Seção 1, p. 8-11. Disponível em: https://faceres.com.br/cursos/medicina/diretrizes-curriculares-nacionais-medicina-de-2014

4. Ribeiro VMB. O porquê e o para quê de um seminário sobre formação pedagógica de preceptores: situando o Projeto. In: Ribeiro VMB, organizador. Formação pedagógica de preceptores do ensino em saúde. Juiz de Fora: UFJF; 2011 [citado em 2016 Abr 4]. p. 5-10. Disponível em: http://www.editoraufjf.com.br/ftpeditora/site/formacao_pedagogica_de_preceptores_ do_ensi no_em_saude.pdf

5. Feuerwerker LCM, Merhy EE. A contribuição da atenção domiciliar para a configuração de redes substitutivas de saúde: desinstitucionalização e transformação de práticas. Rev Panam Salud Publica/Pan Am J Public Health [Internet]. 2008 [citado em 2016 Abr 22];24(3):180-88. Disponível em: http://www.scielosp.org/pdf/rpsp/v24n3/a04v24n3.pdf

6. Skare TL. Metodologia do ensino na preceptoria da residência médica. Revista do Médico Residente. 2012;4(2):116-20.

7. Marin MJS, Lima EFG, Paviotti AB, et al. Aspectos das fortalezas e fragilidades no uso das metodologias ativas de aprendizagem. Rev Bras Educ Med. 2010;34(1):13-20. http://dx.doi.org/10.1590/S0100-55022010000100003.

8. Turato ER. Tratado da metodologia da pesquisa clínico-qualitativa. Petrópolis: Vozes; 2003.

9. Gil AC. Métodos e técnicas de pesquisa social. 6. ed. São Paulo: Atlas; 2008.

10. Brasil. Ministério da Educação. Secretaria de Educação Superior. Comissão Nacional de Residências Medica. Resolução CNRM n ${ }^{\circ}$ 02/2006, de 17 de maio de 2006. Dispõe sobre requisitos mínimos dos Programas de Residência Médica e dá outras providências. Diário Oficial da União, Brasília, DF, n. 95, 2006. Seção 1, p. 23-36.

11. Minayo MC. Análise qualitativa: teoria, passos e fidedignidade. Cien Saude Colet. 2012;17(3):621-6. http://dx.doi.org/10.1590/ S1413-81232012000300007. PMid:22450402.

12. Bardin L. Análise de conteúdo. Lisboa: Edições 70; 2009.

13. Botti SHO. O Papel do Preceptor na Formação de Médicos Residentes: um estudo de residências em especialidades clínicas de um hospital de ensino [tese]. Rio de Janeiro: Escola Nacional de Saúde Pública Sérgio Arouca; 2009 [citado em 2016 Abr 12]. 144 p. Disponível em: http://www.arca.fiocruz.br/bitstream/icict/2582/1/ENSP_Tese_Botti_Sergio_Henrique.ppd

14. Cadernos da ABEM. O preceptor por ele mesmo [Internet]. Vol. 9. Rio de Janeiro: Associação Brasileira de Educação Médica; 2013 [citado em 2016 Maio 5]. Disponível em: http://www.abem-educmed.org.br/pdf/cadernos_vol_8.pdf

15. Brasil. Ministério da Saúde. Agência Nacional de Vigilância Sanitária. Resolução - RDC nº 36 , de 25 de julho de 2013 . Institui ações para a segurança do paciente em serviços de saúde e dá outras providências. Diário Oficial da União [Internet]. Brasília, 


\section{ijhe}

2013 [citado em 2019 Out 2]. Disponível em: http://bvsms.saude.gov.br/bvs/saudelegis/anvisa/2013/rdc0036_25_07_2013. htmln

16. Berbel NAN. A metodologia da problematização em três versões no contexto da didática e da formação de professores. Revista Diálogo Educacional. 2012;12(35):103-20.

17. Guimarães SER. Avaliação do estilo motivacional do professor: adaptação e validação de um instrumento [tese]. Campinas (SP): Programa de Pós-graduação em Educação, Faculdade de Educação, Universidade Estadual de Campinas; 2003. 189 p.

18. Lima VV, Padilha RQ, Olivera SO, Pereira SMSF. Processos educacionais na saúde: aperfeiçoamento com ênfase na aprendizagem significativa. São Paulo: Instituto Sírio-Libanês de Ensino e Pesquisas; 2015.

19. Araújo SHR. Análise de produção científica brasileira sobre as metodologias ativas de aprendizagem na área de saúde [dissertação]. Maceió (AL): Programa de Pós-graduação em Ensino e Saúde, Faculdade de Medicina, Universidade Federal de Alagoas; 2013. 43 p.

20. Rocha HM, Lemos WM. Metodologias ativas: do que estamos falando? Base conceitual e relato de pesquisa em andamento. In: Anais do IX SIMPED - Simpósio Pedagógico e Pesquisas em Educação; 2014; Resende, RJ. Resende (RJ): AEDB.

21. Sebold LF, Martins FE, Da Rosa R, Carraro TE, Martini JG, Kempfer SS. Metodologias ativas: uma inovação na disciplina de fundamentos para o cuidado profissional de enfermagem. Cogitar e Enfermagem. 2010;15(4):753-6. http://dx.doi. org/10.5380/ce.v15i4.20381.

22. Oliveira EM, Ferreira WFS. Dilemas no processo de auditoria: paradoxos dos serviços em saúde. Rev Univ Vale Rio Verde. 2016;14(2):659-77.

\section{Contribuição dos autores}

Vilma Francisca Hutim Gondim de Souza participou da elaboração do projeto, coleta de dados, transcrição e análise das entrevistas e confecção do manuscrito. Lizomar de Jesus Maués Pereira Moia participou da concepção inicial, acompanhamento, orientação do estudo e elaboração do manuscrito. Ingrid Magali de Souza Pimentel participou da concepção do projeto, acompanhamento, orientação do estudo e elaboração do manuscrito. 